\title{
Perspectivas docentes sobre la formación de competencias investigativas en relación con los programas de asignatura.
}

\section{Teachins perspectives on the investigative competences training in relation to subjet programs.}

\section{Esta obra está bajo una Licencia Creative Commons Atribución 4.0 Internacional.} DOI: https://doi.org/10.32870/sincronia.axxii.n74.29b18

\section{Cuauhtémoc Banderas Martínez}

Departamento de Letras / Universidad de Guadalajara

cbanderas2003@hotmail.com

(MÉXICO)

\section{Gabino Cárdenas Olivares}

Departamento de Letras / Universidad de Guadalajara

gabinocardenas@gmail.com

(MÉXICO)

\section{María Estela Martínez Castro}

Departamento de Estudios de la Comunicación Social / Universidad de Guadalajara estelamartinezmx@yahoo.com.mx

(MÉXICO)

Recibido: $14 / 03 / 2018$

Revisado: 15/03/2018

Aprobado: $12 / 04 / 2018$

\section{RESUMEN}

La formación para la investigación es un reto para los académicos del área de metodología en los programas académicos de educación superior. La comunicación entre los docentes es indispensable si se pretende un trabajo colaborativo en la formación y desarrollo de competencias investigativas en los estudiantes. La formación de este tipo de competencias se logra necesariamente en el campo de la investigación, ya sea acompañando a los estudiantes en la realización de investigaciones propias de ellos o dirigiendo la elaboración de las tesis para la obtención del grado académico. Las 
perspectivas de los docentes sobre los aspectos que consideran en la enseñanza para la investigación son clave para entender las características tanto del trabajo individual como del trabajo colegiado en relación, en lo general, con los programas de asignaturas del plan de estudios de una licenciatura, y en lo particular, en el campo de la metodología de la investigación. La contribución de los estudios universitarios a la solución de los problemas del entorno parte de involucrar a los estudiantes en la realización de investigaciones en el campo de su formación profesional.

En este trabajo exponemos los aspectos clave que los docentes consideran sobre su quehacer académico para la formación de competencias investigativas.

Palabras clave: Competencias. Investigación. Educación Superior. Comunicación entre docentes. Formación profesional.

\section{ABSTRACT}

The training for investigation is a challenge for the methodology area teachers on the higher education academic programs. The communication among the teachers is essential if seeking a collaborative work on the training and development of the investigative competences in students. The training on this type of competences is necessarily achieved on the investigation field, by accompanying the students during the conduction of their own investigations or leading the elaboration of theirs thesis to obtain an academic degree. The teachers perspectives about the aspects that they consider during the teaching for investigation are fundamental to understand the characteristics both on individual projects as on collegial projects related, in general, to the subject programs of a bachelor's degree syllabus, and, particularly, on the investigation methodology field. The contribution of university studies to the solution of environment problems starts with involving the students on the conduction and elaboration of investigations related to their vocational training field. On this work, we expose the key aspects that the teachers consider during their academic chore for the training of investigative competences.

Keywords: Competences. Investigation. Higher education. Communication among teachers. Vocational training.

\section{Formación para la investigación}

La formación académica basada en competencias trasciende las habilidades y el conocimiento al incluir elementos actitudinales y axiológicos observables y medibles en la ejecución. Esta tendencia educativa internacional propició la precisión de las competencias clave en los currículos, como ha sucedido en la educación obligatoria de la mayoría de los países europeos (Núñez, 2016).

La educación superior tiene tres funciones sustantivas: docencia, investigación y difusión. Las dos primeras están encaminadas directamente a la formación profesional, en tanto que la tercera 
proyecta a la sociedad el conocimiento que se genera en ella. También, la educación superior forma al profesionista en el interés por su participación cívica y social (Llano, 2003). Ante las funciones sustantivas de la universidad se mantienen dos posturas: una crítica y otra participativa. La crítica cuestiona y mueve desde los planteamientos racionales, la participativa mueve desde la acción. Esto es así, porque la institución de educación superior no puede conformarse con la mera transmisión de saberes, sino que se reconoce formadora de profesionales y de ciudadanos que solucionen los problemas del entorno e innoven las prácticas en los contextos particulares de actuación (Navío, 2005). La formación académica con base en el desarrollo de competencias parte de un cuestionamiento crítico de la realidad natural y social que permite a los individuos indagar y responder a ella de manera compleja en la intervención para la solución de las problemáticas que los circunscriben.

Si se opta por las competencias en los currículos de formación profesional y, por ende, en los programas de asignatura, se asume la capacidad de aplicar conocimientos y destrezas facilitando las transferencias y el aprendizaje permanente (Montero, 2009), así como del grado de eficiencia de la aplicación de las competencias en situaciones reales en la evaluación de los estudiantes (Zabala y Arnau, 2008). De esta manera, la enseñanza basada en el desarrollo de competencias requiere criterios de evaluación acordes con aprendizajes aplicados, como las rúbricas que explicitan criterios de desempeño o niveles de dominio sobre ellos.

En México, la formación de competencias investigativas en pregrado se ha incrementado notablemente a partir de 2007, debido a la necesidad de formación profesional en la generación del conocimiento y su vínculo con las demandas de la sociedad. El desarrollo de la investigación ha propiciado la solidez de las estructuras universitarias para cumplir con esa función sustantiva de la universidad y el repunte de la misma investigación desde la formación profesional de pregrado. Esto porque la universidad no puede entenderse sin la investigación y ésta es una función clave del profesorado en cuanto sector académico docente $y$, en consecuencia, de los estudiantes en cuanto profesionales en formación. 
Benavides Lara (2017) sostiene que entre los años 2007 y 2013, el número de docentes que laboraban en las 45 principales universidades de la República Mexicana aumentó un 14.9\%, en tanto que el de los investigadores en las mismas universidades se incrementó en un $40.2 \%$ El mismo autor, con base en Cummings y Cheol (2014) hace notar que el interés de los profesores universitarios por la investigación es mayor que su interés por la enseñanza, fenómeno que también ocurre en universidades de Brasil, China, Sudáfrica y Malasia. Es de suponerse que este interés docente por la investigación impactará la formación profesional de los estudiantes mediante diversas actividades investigativas en la formación universitaria de pregrado.

La enseñanza de la investigación está lejos de concebirse como uniforme y lineal. Sánchez Puentes (2014, p. 11) sostiene que "enseñar a investigar es un proceso complejo y una actividad diversificada." Se puede considerar que en la formación de competencias investigativas se conjuga el oficio del investigador y su función pedagógica. Quien investiga puede enseñar a investigar. El aprendiz puede investigar y utilizar esta práctica como recurso de aprendizaje. Guadarrama González (2014, p. 25) pone el dedo en la herida, al reconocer que "es lamentable que en ocasiones algunos profesores de metodología de la investigación científica no han sido ellos personalmente ejecutores de algún proyecto de investigación, o no hayan elaborado o sustentado alguna tesis de maestría o doctorado". En este sentido, es presumible que sólo puede enseñar a investigar quien investiga, sin embargo se reitera que el proceso de enseñanza y aprendizaje no es lineal, porque entran en juego tanto las competencias didácticas de quien enseña como las habilidades de quien aprende. Qué tan bien se enseñe y/o se aprenda a investigar encierra una complejidad mayor.

Se entiende que la enseñanza y el aprendizaje son procesos dialécticos complejos. Dialécticos en sí mismos. Complejos respecto del conjunto de factores que lo impactan simultáneamente, "por lo mismo, no hay esquemas o modelos de investigación únicos y definitivos, sino sólo guías que orientan el desarrollo del trabajo de investigación, las cuales se ajustan a los requerimientos que exige la práctica científica en cada situación concreta" (Rojas, 2001, p. 93). Se reitera, no existen procesos lineales en la formación de competencias investigativas ni recetas que 
se apliquen para garantizar al cien por ciento el desarrollo de las mismas en los aprendices. Lo mismo ocurre en cualquier otro tipo de aprendizaje: procesos dialécticos y complejos en los que lo más natural es que se avance y se retroceda, se replanteen posturas y se generen dudas, se asuman certezas y se desmoronen enseguida como necesidad de generación del conocimiento.

Toda investigación ha de contener los fundamentos epistemológicos y claridad en los componentes que la constituyen, como cuestionamientos sobre la realidad, alcances, objetivos e hipótesis, métodos de observación, validación y confiabilidad de los instrumentos para obtener la información, sus propios análisis e interpretaciones, sus heurísticas y sus explicaciones. No existen dos investigaciones idénticas. Los estudiantes lo deben saber. "La acción educativa es un proceso vivencial en un recorrido cíclico, a manera de espiral, que abarca las dimensiones referidas a lugares, objetos, actores y actividades. Esto es, qué hacen las personas, con qué y donde", apuntan Muñoz Giraldo, Quintero Corzo y Múnevar Molina (2014, p. 17). Los profesores han de propiciar en sus alumnos la seguridad de la incertidumbre en la inseguridad de la certidumbre, el camino heurístico e incierto de la investigación, la exigencia del rigor científico y el disfrute del trabajo investigativo.

Es verdad que en el aula se puede enseñar y aprender a investigar en el aula de manera conceptual mediante la consulta de bibliografía, pero nada supera la acción para cumplir este propósito que desarrollar competencias investigativas investigando, ya sea como responsable de un proyecto o como asistente en una investigación o, bien, dirigiendo una tesis de grado y aprender a investigar siendo dirigido en el acto mismo de investigar para generar el conocimiento que se pretende. Así, las tesis de grado -en cuanto reportes de investigaciones realizadas a defenderse para la obtención de un grado académico y que presumen cumplir con el rigor metodológico al menos suficiente- pueden servir como referente de análisis y verificación del desarrollo de dominio de competencias investigativas.

\section{Formación de competencias investigativas}


La formación de competencias investigativas implica obtener conocimientos sobre los procesos de investigación, desarrollar habilidades metodológicas, disponer actitudes de interés, admiración y disciplina, así como mantener valores de honestidad y respeto, además del reconocimiento al trabajo de terceros. Con la expresión "competencias investigativas" se hace referencia a un conjunto de habilidades de diversa naturaleza en el campo de la investigación, las cuales empiezan a desarrollarse desde antes de que el individuo tenga acceso a procesos sistemáticos de formación para la investigación, que en su mayoría no se desarrollan sólo para posibilitar la realización de las tareas propias de la investigación académica sino para la adquisición de saberes y solución de porblemas (Moreno, 2005). De acuerdo con esta investigadora la formación de estas competencias debe permearse durante todo el proceso formativo y atenderse en todos los niveles educativos, en forma de un curriculun transversal. La cuestión fundamental a resolver es la especificación de cuáles son estas competencias y cuál es el grado de desarrollo que debe lograse en cada nivel educativo.

Queda claro que el nivel de doctorado es el espacio en el cual se forman los investigadores y que los egresados de este nivel educativo cuentan con las competencias requeridas para generar conocimiento; sin embargo, esta formación para la investigación no está del todo presente en los niveles de maestría y licenciatura. Hasta hace pocos años, era condición que para obtener el grado de maestro se presentara un trabajo de investigación (tesis) y se defendiera ante un jurado, pero actualmente ya no ocurre en todos los programas de maestría, principalmente debido a la exigencia que padecen las universidades para elevar los índices de eficiencia terminal; esto mismo ocurre en el nivel de licenciatura, donde la tesis es sólo una de las modalidades de titulación y ya no es la única. ¿Quién pierde y qué se pierde con la eliminación de la exigencia de un trabajo de investigación para la obtención del grado académico en los programas de licenciatura y maestría? El problema es que no hay criterios bien definidos sobre la calidad de los trabajos que se requiere en cada nivel. Se asume que lo que se exige en un doctorado es de mayor rigor que lo que se pide en el nivel de maestría y licenciatura, por lo que se infiere que los profesores de pregrado difícilmente saben con precisión cuáles son las competencias investigativas que deben formar ni 
cuál es el nivel de desarrollo de una investigación al que deben aspirar con sus estudiantes tanto en extensión como en profundidad, principalmente si no existe comunicación entre los docentes del área de metodología ni articulación entre los programas de asignatura respectivos.

Al revisar los programas de asignatura de la licenciatura en Letras hispánicas, de la Universidad de Guadalajara, se percata de que en ellos no se describen las competencias investigativas que propiciarán los profesores para desarrollar en los estudiantes. Lo mismo sucede en el Plan de estudios donde se establece que el egresado: "Posee un acervo intelectual de herramientas teórico-metodológicas para realizar investigación lingüística y literaria en el ámbito de las diferentes manifestaciones de la lengua española, [competencias] para proponer proyectos académicos con base en propuestas de análisis...", lo cual evidentemente no es una descripción de la competencia.

En los programas de asignatura existe un apartado denominado Unidad de competencia en el que se supone debería definirse, precisamente, el nivel de logro de las competencias. Sin embargo, en los programas de asignatura los profesores no especifican si se refiere a una competencia en particular o a todas las competencias propuestas en el Plan de estudios. Es un hecho que los programas de asignatura mantienen relaciones de diversa naturaleza con las competencias, y podemos suponer que todas las asignaturas de la academia de metodología deben contribuir de una u otra manera al desarrollo de las competencias investigativas. Cierto es que la mayoría de estas asignaturas se refieren al análisis y a la crítica literaria, pero también podemos pensar que el análisis y la crítica literaria son formas particulares de investigación.

Los supuestos mencionados en estas líneas nos dan la pauta para interpretar en las respuestas dadas por los docentes ante preguntas específicas sobre la formación o desarrollo de competencias investigativas en los estudiantes de licenciatura. De principio, podemos separar las respuestas en dos grupos: en el primero, agrupamos aquellas que se centran en el desarrollo de habilidades propias de la investigación, las cuales mencionan la investigación documental, la elaboración de protocolos, el uso de metodologías, la elaboración de hipótesis, etc., y en el 
segundo, aquellas respuestas que están en función de los objetivos y los contenidos propios de las unidades de aprendizaje que imparten los docentes.

Uno de los supuestos básicos de esta investigación es que el nivel de licenciatura o pregrado no es considerado como el espacio idóneo para formar investigadores, ya que este papel se les ha asignado a los posgrados y que el éxito de estos depende en mayor o menor medida de que el aspirante a investigador cuente con una sólida y bien estructurada formación disciplinar y que haya desarrollado las competencias investigativas desde la licenciatura.

Uno de los objetivos de la investigación a la que se refiere este artículo fue constatar si las competencias investigativas constituyen un eje primordial de la formación académica de los alumnos de Licenciatura en Letras Hispánicas de la Universidad de Guadalajara. Para ello se hizo necesario indagar el nivel de desarrollo de las competencias investigativas en los estudiantes, así como la efectividad de las prácticas educativas para este fin, y, de esta manera indagar cuáles son los aspectos del proceso de investigación que hace falta reforzar para que el estudiante comprenda y domine la totalidad del proceso investigativo. Aquí sólo se muestran las perspectivas de los docentes sobre la formación de competencias investigativas.

Son muchos los factores que impactan el proceso formativo en las universidades, algunos de forma más o menos directa o inmediata. Entre estos se pueden considerar las políticas educativas, tanto a nivel general como a nivel institucional, las necesidades y demandas de los diversos sectores sociales, el financiamiento, los modelos educativos, los planes de estudio, la formación académica y la calidad de los docentes, así como las condiciones específicas en las que se desarrollan las actividades de enseñanza aprendizaje o prácticas educativas.

De manera específica, en este espacio interesa destacar dos aspectos fundamentales, a saber, los planes y programas de estudios y las prácticas educativas a través de los cuales estos se concretan. Se reconoce que los docentes constituyen el factor decisivo en este proceso, pues son ellos quienes materializan cotidianamente en el aula la propuesta formativa del currículo. 
Además, se considera que las competencias investigativas son el eje fundamental de la formación de los estudiantes de la licenciatura en Letras Hispánicas, ya que son la base de todas las actividades que se supone podrá desempeñar un egresado de esta licenciatura, así, por ejemplo, para poder desarrollar propuestas de formación en lengua y literatura, hacer crítica y análisis literario o formular programas de difusión cultural, protocolos para la investigación en lengua y literatura, requiere de realizar investigación, ya que en todas estas actividades es necesaria.

En el análisis de una muestra de veinte programas de asignatura del Área de Formación Básica Obligatoria del plan de estudios de la licenciatura mencionada se encontró que dichos programas siguen manteniendo los esquemas educativos de los modelos tradicionales, pues en ellos se conserva un carácter más informativo que formativo de la enseñanza, al enfatizar la transmisión y asimilación de contenidos disciplinares más que el desarrollo de las habilidades específicas para la investigación.

En consecuencia, difícilmente se puede lograr uno de los principales propósitos del proyecto curricular, a saber: lograr que los egresados adquieran una la formación disciplinar y metodológica que los haga competentes para realizar investigación en los ámbitos de la lengua, la literatura y la docencia; esto se debe a que las actividades de investigación -investigación formativa en este caso- no forman parte de los procesos y saberes contemplados en los programas de asignatura y, consecuentemente, no están integrados de manera sistemática al proyecto curricular (Véase Banderas, Cárdenas, Martínez, 2013).

Los datos obtenidos en el análisis realizado muestran que existen programas en los que se exige presentar un trabajo (ensayo, análisis u otro) cuyo proceso de elaboración no forma parte, al menos no de manera explícita, de los contenidos curriculares del programa de asignatura, con lo cual existe una alta posibilidad de que tampoco formen parte del proceso de enseñanza y aprendizaje (Banderas, Cárdenas, Martínez, 2013). Este es un problema que puede subsanarse con base en las perspectivas de los docentes, reflejadas en el discurso acerca del ejercicio particular de su docencia. 


\section{Análisis de resultados}

Con base en el antecedente expuesto renglones arribas se planteó la necesidad de acercarse más al mundo de las prácticas de enseñanza y aprendizaje y ante las dificultades que implica irrumpir en la intimidad de las aulas a través de la observación directa de las actividades, se decidió aplicar un cuestionario a los profesores que imparten las asignaturas que conforman la Academia de metodología, por ser estas las que están más directamente relacionadas con la formación de las competencias investigativas.

El cuestionario se aplicó a nueve profesores, los cuales imparten las siguientes asignaturas: Introducción a la crítica literaria, Seminario de titulación en Letras hispánicas, Teoría del análisis literario, Semiótica, Análisis del discurso, Teoría literaria, Fundamentos del análisis literario, Metodología de la investigación literaria y poética.

Las preguntas que se les hicieron estuvieron dirigidas a conocer algunos aspectos que tienen que ver con la manera en la que a través de su trabajo en el aula se fomenta el desarrollo de las competencias. Las preguntas incluyen, además de la propia conceptualización de las competencias, indagar sobre las fortalezas y debilidades que con base en la práctica educativa de los profesores presentan los estudiantes en este rubro, así como preguntas relacionadas con las actividades respecto de la investigación, el apoyo y el seguimiento que proporcionan a los alumnos al respecto, así como el nivel de desarrollo que logran los estudiantes, entre otras.

A continuación se exponen las preguntas y parte del análisis realizado.

\section{Pregunta uno:}

Como docente del área de metodología en lengua y literatura, ¿cómo define usted las competencias investigativas?

\begin{tabular}{|c|c|}
\hline Informante & RESPUESTAS \\
\hline 1 & "son esenciales para generar reflexión mayor acerca del objeto de \\
& estudio literario... investigar, documentarse, discriminar información, \\
\hline
\end{tabular}




\begin{tabular}{|c|c|}
\hline & reflexionarla y aplicarla." \\
\hline 2 & $\begin{array}{l}\text { "capacidades, habilidades y actitudes...que desarrolla una persona para } \\
\text { resolver problemas de investigación en lengua y literatura." }\end{array}$ \\
\hline 3 & $\begin{array}{l}\text { "la capacidad de indagar a profundidad sobre un tema... capacidad de } \\
\text { localizar y seleccionar material adecuado... sintetizar las ideas y ser capaz } \\
\text { de transmitir de manera coherente y sólida la información." }\end{array}$ \\
\hline 4 & $\begin{array}{l}\text { "la identificación, formulación y resolución de problemas... la generación } \\
\text { y difusión del conocimiento..." }\end{array}$ \\
\hline 5 & $\begin{array}{l}\text { "...sabrá analizar los textos literarios, académicos y/o culturales } \\
\text { correspondientes en general." }\end{array}$ \\
\hline 6 & $\begin{array}{l}\text { "principalmente por saber acceder a las bibliografías en la crítica } \\
\text { literaria." }\end{array}$ \\
\hline 7 & $\begin{array}{l}\text { "habilidad para cuestionarse sobre el porqué o para qué de los hechos o } \\
\text { fenómenos...la decisión para llevar a cabo una búsqueda propia y } \\
\text { original... consultar material de diverso tipo, saber seleccionar la } \\
\text { información relevante, registrarla y organizarla para sustentar con ella su } \\
\text { trabajo." }\end{array}$ \\
\hline 8 & $\begin{array}{l}\text { "La formación de estudiantes...para conocer a través del uso de } \\
\text { estrategias metodológicas para hacer investigación literaria." }\end{array}$ \\
\hline 9 & $\begin{array}{l}\text { "...conjunto de conocimientos, habilidades y actitudes que se aplican en } \\
\text { el desempeño de una función productiva o académica" }\end{array}$ \\
\hline
\end{tabular}

Con excepción de la respuesta del informante 2, que es una definición muy genérica de las competencias, las otras respuestas de los profesores, como se puede observar en los fragmentos seleccionados, no son, en sentido estricto, definiciones de las competencias en cuestión; algunas respuestas destacan su función e importancia (informante 1), otras, enumeran las capacidades o aptitudes que implica el desarrollo de estas competencias, así, un individuo competente deberá saber identificar, formular y solucionar un problema de investigación (informantes 1, 3, 4 y 7 ); además deberá tener la capacidad para seleccionar la información adecuada, consultar material de 
diverso tipo, registrarla y organizarla para sustentar con ella su trabajo (1, 3 y 7$)$, y para sinterizar y transmitir coherentemente información (3).

Resaltan, por sus particularidades, la siguientes respuestas: la del informante 5 , que reduce la competencia a la capacidad para analizar textos; la respuesta del informante 6, que etiqueta la capacidad de acceder al ámbito exclusivo de la bibliografía de la crítica literaria, y las respuestas de los informantes 8 y 9, que hacen alusión al aspecto formativo de las competencias y su aplicación en diversos ámbitos.

\section{Pregunta dos:}

¿Hace usted una evaluación diagnóstica sobre el nivel de dominio de competencias investigativas de sus alumnos al inicio de su curso?

\begin{tabular}{|c|c|}
\hline Informante & RESPUESTAS \\
\hline 1 & $\begin{array}{l}\text { Realizo una actividad inicial para "evaluar el nivel de crítica literaria } \\
\text { que tienen los estudiantes..." }\end{array}$ \\
\hline 2 & $\begin{array}{l}\text { Diagnóstico grupal sobre "qué saben acerca de los componentes de } \\
\text { un protocolo de investigación y cuál es su disposición ante la } \\
\text { investigación." }\end{array}$ \\
\hline 3 & “No" \\
\hline 4 & “No" \\
\hline 5 & $\begin{array}{l}\text { Sondeo para detectar "Ios vicios y los errores de aplicación de las } \\
\text { herramientas metodológicas." }\end{array}$ \\
\hline 6 & “No. Pero una breve evaluación en habilidades de lectura sería útil”. \\
\hline 7 & $\begin{array}{l}\text { Hablar "sobre los trabajos de investigación llevados a cabo en } \\
\text { semestres anteriores." }\end{array}$ \\
\hline 8 & $\begin{array}{l}\text { "Diagnóstico mediante preguntas orales sobre su experiencia e } \\
\text { intereses, así como <<la percepción que tiene referente a la práctica } \\
\text { de la investigación literaria>>." }\end{array}$ \\
\hline 9 & "Por medio de una lectura de un texto narrativo les demuestro que \\
\hline
\end{tabular}


son leedores y no lectores."

Con excepción de los informantes 3,4 y 6, todos los demás realizan algún tipo de diagnóstico sobre las competencias investigativas, el cual sondea sobre sus experiencias en la investigación, especialmente en la elaboración de protocolos y en la aplicación de metodologías. Llama la atención la respuesta del informante 1, que evalúa el "nivel de crítica literaria", y el informante 9 que evalúa las habilidades lectoras de los estudiantes para demostrarles "que son leedores y no lectores."; esta misma referencia a las habilidades lectoras es sugerida por el informante 6, el cual, aunque acepta no hacer ningún tipo de diagnóstico, propone evaluar la capacidad para la lectura.

\section{Pregunta tres:}

¿Cuáles son los aspectos más débiles que encuentra en sus alumnos respecto de las competencias investigativas?

\begin{tabular}{|c|l|}
\hline Informante & \multicolumn{1}{|c|}{ RESPUESTAS } \\
\hline 1 & $\begin{array}{l}\text { "Considero que la mayor debilidad reside en la } \\
\text { discriminación de la información... no hay un momento de } \\
\text { 'procesamiento' de la información...falta el análisis, falta el } \\
\text { procesamiento de información." }\end{array}$ \\
\hline 2 & $\begin{array}{l}\text { "El ver la investigación como un todo coherente...algo de } \\
\text { inseguridad sobre sus conocimientos previos." }\end{array}$ \\
\hline 3 & "La falta de conocimientos para la elaboración de hipótesis." \\
\hline 4 & $\begin{array}{l}\text { "No identifican situaciones problemáticas, no formulan, } \\
\text { no generan soluciones." }\end{array}$ \\
\hline 5 & $\begin{array}{l}\text { "Su Capacidad para el estudio de conceptos teóricos y } \\
\text { implica el trabajo de investigación." }\end{array}$ \\
\hline
\end{tabular}




\begin{tabular}{|c|c|}
\hline & previamente publicada en sus propios trabajos." \\
\hline 7 & $\begin{array}{l}\text { "Pensar que en internet se encuentra toda la } \\
\text { información...No cuestionarse si la información que } \\
\text { encuentran en la red es verídica o suficiente. Desconocer } \\
\text { sitios a dónde acudir en busca de información } \\
\text { documental,...No tener el hábito de comparar la información } \\
\text { que proporcionan las diversas fuentes o autores, suelen } \\
\text { limitarse a citar solamente a uno. Pensar que la única fuente } \\
\text { de consulta (además de internet) consiste en libros, sin } \\
\text { considerar otro tipo de textos: publicaciones periódicas, } \\
\text { fotografías, mapas, cartas, etc." }\end{array}$ \\
\hline 8 & "Expresar sus ideas de forma oral." \\
\hline 9 & $\begin{array}{l}\text { "Cuando lee el alumno envuelve el texto en un tema, teoría } \\
\text { o noción predeterminada para ajustar al texto a sus } \\
\text { esquemas (prejuicios) del lector, estrategia de 'dictador' } \\
\text { (Deducción). No hay diálogo con el texto, lo sujeta. Dialogar } \\
\text { de manera a priori a nociones preestablecidas." }\end{array}$ \\
\hline
\end{tabular}

La lista de deficiencias detectadas por los profesores es larga y muy significativa, nos da una idea cercana del nivel que tienen los estudiantes en el rubro de las competencias investigativas. Destacan las respuestas que mencionan las relacionadas con la búsqueda y selección de la información y la incapacidad para diversificar la búsqueda de fuentes de información y para seleccionar la más adecuada (informantes 1 y 7), así como la incapacidad para definir un problema de investigación y ver la investigación como un todo (informantes 2 y 4 ).

Llaman la atención la respuesta del informante 5, que resalta falta de comprensión de conceptos teóricos, y la del informante 6, que menciona la falta de conocimientos para elaborar hipótesis. Estas deficiencias son muy significativas; una persona que sabe leer, además de comprender, puede analizar, criticar, y como consecuencia, es capaz de utilizar la teoría para definir y fundamentar una investigación, así como elaborar los marcos teóricos y fundamentar las 
hipótesis, así mismo, podríamos pensar que la incapacidad para expresar las ideas en forma oral, mencionada por informante 8 , tiene su origen en estas deficiencias. Por ello es conveniente reflexionar sobre esto, pues no es posible que alguien que presenta deficiencias en sus capacidades lectoras pueda ser un investigador competente.

La respuesta del informante 9 se centra de manera particular en resaltar el fomento a las competencias lectoras y en considerar la lectura como una de las herramientas fundamentales para el análisis de textos, particularmente de textos literarios; una lectura enmarcada en conceptos teóricos que le dan sentido al acto mismo de leer.

\section{Pregunta cuatro:}

¿En su clase los estudiantes realizan actividades de investigación?

\begin{tabular}{|c|c|}
\hline Informante & RESPUESTAS \\
\hline 1 & $\begin{array}{l}\text { "...les solicito que consideren el objeto de estudio sobre el que } \\
\text { realizarán su análisis final expresado en un ensayo académico.... } \\
\text { Investigan una teoría o corriente crítica literaria." }\end{array}$ \\
\hline 2 & $\begin{array}{l}\text { "...elaboran un proyecto en cada uno de sus componentes... } \\
\text { (elaboración de un protocolo completo)" }\end{array}$ \\
\hline 3 & $\begin{array}{l}\text { "Primero les expongo procedimientos para detectar, delimitar y } \\
\text { formular problemas de investigación, luego redactamos textos } \\
\text { académicos e investigativos..." }\end{array}$ \\
\hline 4 & $\begin{array}{l}\text { "Sí, en dos momentos. Primero... investigación sobre ciertos conceptos } \\
\text { que deben saber y no conocen... El segundo cuando terminan su } \\
\text { análisis textual...investigar contextos sociales que generan las } \\
\text { problemáticas sociales del texto." }\end{array}$ \\
\hline 5 & "Sí, hacemos resúmenes de varios artículos críticos" \\
\hline 6 & $\begin{array}{l}\text { Sí, que “...conozcan la ubicación y el acervo las } 20 \text { principales } \\
\text { bibliotecas y archivos que existen en la Zona Metropolitana de } \\
\text { Guadalajara. Visitar esos acerbos y entregar un reporte. Además, }\end{array}$ \\
\hline
\end{tabular}




\begin{tabular}{|c|l|}
\hline & considerar si se requiere de investigación de campo." \\
\hline 7 & $\begin{array}{l}\text { "Redacción de un protocolo. Elaboración de estudio de caso y reportes } \\
\text { de investigación literaria. Diseño de encuestas o guías para realizar } \\
\text { entrevistas...Presentación y exposición de avances de investigación." }\end{array}$ \\
\hline 8 & $\begin{array}{l}\text { "Redacción de un protocolo. Elaboración de estudio de caso y reportes } \\
\text { de investigación literaria. Presentación de avances por cada grupo de } \\
\text { estudio. Exponer los resultados de investigación colectiva. Redactar un } \\
\text { reporte de investigación..." }\end{array}$ \\
\hline 9 & $\begin{array}{l}\text { "...Solo se hacen lecturas para aplicar los conceptos con los cuales se } \\
\text { responde a la pregunta ¿Qué es leer?" }\end{array}$ \\
\hline
\end{tabular}

Todas las respuestas son afirmativas en este sentido: en todas las asignaturas se realizan algunas actividades de investigación. Resaltan las respuestas que mencionan la realización de un protocolo (7 y 8) o proyecto de investigación (2), y otras tienen que ver con la selección, definición y delimitación de un tema de estudio, mencionados por tres informantes (1, 3 y 4).

Las respuestas que llaman la atención por diferentes son las que únicamente indican la realización de actividades de lectura, ya de textos teóricos y/o literarios, cuyo resultado sería un resumen o una reflexión sobre el acto mismo de la lectura $(5,6$ y 9). Si bien es cierto que la lectura es una actividad fundamental para la realización de la actividad investigativa, considerada en sí misma, puede no ser una actividad investigativa, y más aún si no está enmarcada dentro de un proceso investigativo.

\section{Pregunta cinco:}

¿Qué seguimiento da usted a estas actividades?

\begin{tabular}{|c|c|}
\hline Informante & \multicolumn{1}{|c|}{ RESPUESTAS } \\
\hline 1 & “...en el caso de teoría del análisis literario, realizo tres pausas \\
& para solicitar el avance en su análisis: La primera es en la entrega \\
\hline
\end{tabular}


de la definición del objeto de estudio... la segunda para discutir las perspectivas, sugerir bibliografía y apuntalar el trabajo de investigación; el tercero es cuando solicito un avance de su análisis... y exposición de resultados."

“...en la Introducción a la crítica literaria solicito que investiguen

2 por parejas una corriente de crítica literaria...deben compartir con sus compañeros una ficha sintética."

"Acompaño a los estudiantes en la revisión de sus entregas en

3 cada uno de los componentes en relación con su posible tema de investigación...propicio la participación de todos los estudiantes para que retroalimenten a sus compañeros..."

4 “...son raros los alumnos que después de concluir el curso se acercan o continúan la investigación...."

\section{5}

"Planteamiento del problema. Diseño de hipótesis. Delimitación epistémico y teórico. Diseños metodológicos..." etc.

6

"Requiero que los alumnos utilicen los resúmenes como parte de su trabajo."

"a lo largo del semestre van comentando cómo se va desarrollando su proyecto...Al final del curso entregan los 7 reportes escritos sobre las visitas a bibliotecas o archivos, así como su 'bitácora del investigador' donde consignaron todo el proceso..."

"Recomendaciones para fundamentar y argumentar juicios razonamientos; demostrar relaciones entre hechos $y$ argumentos...Aplicar la teoría para comparar semejanzas y diferencias...describir rasgos esenciales y secundarios... expresar criterios propios."

"Evaluación continua de las lecturas de los alumnos." 
De diversas maneras, las respuestas dadas por los informantes evidencian su preocupación porque los estudiantes desarrollen adecuadamente sus trabajos mediante el seguimiento de los procesos de construcción. Las respuestas de los informantes 1, 3, 5 y 7 evidencian un proceso más o menos sistemático y planificado de seguimiento de las actividades desarrolladas por los estudiantes; los demás informantes manifiestan que orientan, sugieren, recomiendan o evalúan, pero no queda claro si esto es periódico, sistemático, regular, o si por el contrario es ocasional fortuito, esto es, no planificado.

Las respuestas de los informantes 2 y 6 no mencionan si hacen o no un seguimiento, mencionan acciones que se les solicitan a los estudiantes. Por último, la respuesta del informante 4, entiende el seguimiento como un proceso que iría más allá del tiempo mismo de la clase.

\section{Pregunta seis:}

En su programa de asignatura o unidad de aprendizaje ¿cuáles competencias investigativas están estipuladas que debe formar?

\begin{tabular}{|c|c|}
\hline Informante & RESPUESTAS \\
\hline 1 & $\begin{array}{l}\text { "Competencia en teoría del análisis literario. Aplicar las herramientas } \\
\text { teórico-metodológicas postestructuralistas para interpretar y valorar } \\
\text { textos, con actitud crítica, honesta y responsable... Competencia en } \\
\text { introducción a la crítica literaria. Poseer un acervo intelectual de } \\
\text { herramientas teórico metodológicas para investigar, interpretar, analizar y } \\
\text { valorar las manifestaciones del fenómeno literario. Competencia en } \\
\text { fundamentos del análisis literario. Dilucidar mensajes cargados de } \\
\text { simbolismo y de relaciones intersubjetivas. Desarrollar habilidades de } \\
\text { orden perceptual, estético y pragmático." }\end{array}$ \\
\hline 2 & $\begin{array}{l}\text { "Elaboración coherente de un proyecto de investigación, sólido en cada } \\
\text { una de sus partes y claramente relacionadas entre si" }\end{array}$ \\
\hline 3 & "La capacidad de dialogar con otras propuestas teóricas" \\
\hline 4 & "No se describen las competencias investigativas". \\
\hline
\end{tabular}




\begin{tabular}{|c|l|}
\hline 5 & $\begin{array}{l}\text { "Desarrolla capacidad de análisis, posee actitud crítica...es honesto y } \\
\text { responsable...comprometido con su desarrollo integral...socializa sus ideas } \\
\text { de forma oral y escrita...es tolerante y respetuoso." }\end{array}$ \\
\hline 6 & $\begin{array}{l}\text { "Que los alumnos puedan responder con argumentos completos a los } \\
\text { argumentos de otros investigadores." }\end{array}$ \\
\hline 7 & $\begin{array}{l}\text { "Habilidades para realizar investigación de tipo documental...localizar la } \\
\text { información...cuestionarla y completarla...organizar esa información, }\end{array}$ \\
\hline 8 & $\begin{array}{l}\text { "Manejo de fuentes documentales...localización de datos... redacción de } \\
\text { textos propios de la investigación." }\end{array}$ \\
\hline 9 & "Reconocer que el concepto actúa como filtro a través del cual ve la \\
& información, le permite seleccionar los hechos o datos, definir el problema \\
\hline
\end{tabular}

Llama la atención la respuesta del informante 4, quien afirma que en los programas de asignatura "no se describen las competencias investigativas". Lo cual es verdad. Lo que existe en ellos es la denominada unidad de competencia que en realidad viene a sustituir el concepto de objetivos que formaban parte de los formatos de programas anteriores con base en cartas descriptivas por objetivos de aprendizaje.

Algunas de las respuestas dadas hacen mención de lo que consignan los programas de asignatura, particularmente en el apartado de los Saberes prácticos, ya que en este se establecen acciones tales como: manejo de fuentes documentales, localización de datos, redacción de textos (7) y elaboración de proyectos de investigación (2). A diferencia de estos, la respuesta del informante 5 menciona lo actitudinal consignado en el apartado de Saberes formativos de los programas de asignatura, y la del informante 1 , que reproduce la unidad de competencia de los programas de asignatura.

Las demás respuestas, al parecer, contienen la visión particular que tiene el profesor de los objetivos que se proponen en el programa de asignatura, tal es el caso de los informante 3 que 
menciona "la capacidad de dialogar con otras propuestas teóricas"; la del 6 que dice que los alumnos "puedan responder con argumentos completos a los argumentos de otros investigadores", y la del 9, que se centra en la función e importancia que tiene "el concepto" en el proceso de lectura y análisis de los textos. Lo común de estas últimas respuestas es que no responden a la pregunta planteada.

\section{Pregunta siete:}

¿Cómo auxilia o apoya a sus estudiantes a que realicen adecuadamente cada elemento de la investigación? ¿Nos puede dar ejemplos?

\begin{tabular}{|c|l||}
\hline Informante & \multicolumn{1}{|c|}{ RESPUESTAS } \\
\hline 1 & $\begin{array}{l}\text { "Asesoro con bibliografía, retroalimento las actividades solicitadas, } \\
\text { pongo a su disposición algunos de los acervos que conozco y en caso de } \\
\text { que necesiten alguna asesoría externa..." }\end{array}$ \\
\hline 2 & $\begin{array}{l}\text { "voy con ellos parte por parte...la consulta a los textos de métodos de } \\
\text { investigación y la retroalimentación que reciben de sus compañeros." }\end{array}$ \\
\hline 3 & $\begin{array}{l}\text { "Les suelo dar una introducción a cómo deben elaborar la hipótesis y la } \\
\text { manera correcta de presentar el trabajo...les pido un borrador de lo } \\
\text { que piensan hacer para retroalimentarlos....Los oriento con bibliografía } \\
\text { oles sugiero que se acerquen con algún colega..." }\end{array}$ \\
\hline 4 & $\begin{array}{l}\text { "Identificar las problemáticas. La delimitación. La formulación del } \\
\text { problema. Establecer criterios teóricos metodológicos. Generar } \\
\text { soluciones. Diseñar proyectos de investigación." }\end{array}$ \\
\hline 5 & $\begin{array}{l}\text { "...después de...aproximarlos a la metodología, pasamos al análisis de } \\
\text { textos. ...comienzan a aplicar las herramientas metodológicas y yo } \\
\text { monitoreo sus aportaciones corrigiendo si es necesario" }\end{array}$ \\
\hline 6 & $\begin{array}{l}\text { "Lo importante es que entiendan que quiero decir por "resumen". } \\
\text { oración) del argumento principal del artículo." }\end{array}$ \\
\hline 5 & "Comparto con ellos experiencias personales que he tenido al realizar \\
\hline
\end{tabular}




\begin{tabular}{|c|l|}
\hline \hline & $\begin{array}{l}\text { investigación, principalmente para mi tesis de maestría y doctorado. ... } \\
\text { Les explico qué tipo de documentos se encuentran en cada una de las } \\
\text { bibliotecas y archivos de la ZMG,... Llevo copia de documentos } \\
\text { originales que he localizado en archivos y hacemos ejercicios de } \\
\text { paleografía... para que se familiaricen con el trabajo de tesis...deben } \\
\text { leer una. Otra actividad que les llama mucho la atención es invitar a un } \\
\text { egresado de la Maestría en Letras de Jalisco..." }\end{array}$ \\
\hline 8 & $\begin{array}{l}\text { "El acompañamiento y seguimiento se orienta a definir y planear la } \\
\text { estrategia metodológica que utilizarán; se realiza un monitoreo } \\
\text { constante en los avances de investigación y se sugieren lecturas que } \\
\text { aporten al tema, todos los integrantes tienen la oportunidad de } \\
\text { aportar observaciones y sugerencias así como formular preguntas." }\end{array}$ \\
\hline 9 & $\begin{array}{l}\text { "Dotándolos de información del concepto con mayor calidad y } \\
\text { pertinencia al tipo de texto que lee." }\end{array}$ \\
\hline
\end{tabular}

Las respuestas, en términos generales, detallan las diversas maneras en que los profesores informantes apoyan el proceso formativo de las competencias investigativas. Así, vemos que asesoran, hacen seguimiento, sugieren materiales de lectura, auxilian en los proceso de formulación de un problema de investigación, elaboración de hipótesis, etc. Todas ellas relacionadas con las actividades de investigación.

La relación de apoyo que relata cada uno de los informantes supone la práctica formativa que realiza para formar las competencias investigativas en sus estudiantes. Se puede considerar que es la parte nuclear de la formación, porque vincula teoría y práctica con el acompañamiento personal o grupal, el cual fundamento para el aprendizaje social.

Las respuestas de los informantes que marcan una pauta diferente son la del informante 6, que afirma "Lo importante es que entiendan que quiero decir por 'resumen'”, y la del 9 que se refiere a dotar de información sobre el concepto más pertinente. En estas respuestas, como se 
puede apreciar, el apoyo brindado a los estudiantes, aparentemente, poco tiene que ver con el desarrollo de las competencias investigativas.

\section{Pregunta ocho:}

¿Cuáles son los elementos que considera más problemáticos en sus alumnos para que comprendan la totalidad del proceso investigativo?

\begin{tabular}{|c|c|}
\hline Informante & RESPUESTAS \\
\hline 1 & $\begin{array}{l}\text { "El tiempo invertido para las actividades. En ocasiones, la parte } \\
\text { teórica no termina por asentarse..." }\end{array}$ \\
\hline 2 & $\begin{array}{l}\text { "Elegir la perspectiva teórica para la investigación...identificar los } \\
\text { principios y categorías de una teoría y cómo utilizarlos en la } \\
\text { interpretación de resultados...elaboración de instrumentos para la } \\
\text { obtención de información." }\end{array}$ \\
\hline 3 & "falta de ideas claras y concretas sobre qué investigar." \\
\hline 4 & $\begin{array}{l}\text { "La identificación, formulación y delimitación de problemas de } \\
\text { investigación." }\end{array}$ \\
\hline 5 & $\begin{array}{l}\text { "Conocimiento y manejo y aplicación de las herramientas } \\
\text { metodológicas para el análisis de textos." }\end{array}$ \\
\hline 6 & $\begin{array}{l}\text { "Convencer a los alumnos de que una lectura cercana es la base para } \\
\text { el trabajo de la crítica literaria." }\end{array}$ \\
\hline 7 & $\begin{array}{l}\text { "con frecuencia comentan que no tuvieron tiempo de seguir } \\
\text { investigando por la premura de entregarlo [el reporte] o porque no } \\
\text { tuvieron tiempo de ir a bibliotecas o no sabían a dónde acudir en } \\
\text { busca de información." }\end{array}$ \\
\hline 8 & $\begin{array}{l}\text { "En algunos casos la elección del tema y en otros definir la } \\
\text { metodología que van a utilizar. El tiempo es su peor enemigo," }\end{array}$ \\
\hline 9 & $\begin{array}{l}\text { "Cuando el alumno intenta ser un lector (y no un leedor) para él es } \\
\text { problemático: observar, asimilar, analizar, comprender e interpretar, } \\
\text { aplicar, transformar y compartir." }\end{array}$ \\
\hline
\end{tabular}


Los aspectos problemáticos mencionados por los informantes en este apartado coinciden en su mayor parte con las deficiencias enumeradas en la pregunta número tres; los tópicos mencionados son: elección y delimitación del tema (4, 3 y 8), conocimiento teórico y aplicación de metodologías $(2,5$ y 8$)$ y la dificultad para comprender los conceptos teóricos, mencionado por los informantes 1 y 2 . Resalta el hecho de que tres informantes $(1,7$ y 8$)$ mencionan la falta de tiempo como un factor problemático en el proceso de investigación, el cual no había sido mencionado en ninguna de las respuestas.

Es de suponer que la identificación de las problemáticas en el proceso de formación de competencias investigativas corresponde al nivel en el que se encuentran los estudiantes respecto del avance en los estudios de la licenciatura. Esto, debido a la variedad de problemáticas reportadas en las respuestas, lo cual puede suponer, además, la posibilidad de la intervención educativa específica para cada problemática detectada por nivel.

\section{Pregunta nueve:}

¿Qué estimado tiene usted de sus avances y logros en el desarrollo de competencias investigativas de sus alumnos?

\begin{tabular}{|c|c|}
\hline Informante & RESPUESTA \\
\hline 1 & "80-85 \%" \\
\hline 2 & $\begin{array}{l}\text { "organizo un coloquio de proyectos de investigación para que los todos los } \\
\text { estudiantes del curso expongan el suyo ante el público asistente". }\end{array}$ \\
\hline 3 & $\begin{array}{l}\text { "...tienen mayor claridad de qué es realizar o elaborar un texto académico } \\
\text { basado en la investigación." }\end{array}$ \\
\hline 4 & "Limitados, sólo algunos casos de tesis." \\
\hline 5 & "entre un 70 u 80 por ciento respecto a cómo llegaron al curso." \\
\hline 6 & $\begin{array}{l}\text { "Aproximadamente } 40 \% \text { de mis alumnos agarran la onda, y hacen intentos } \\
\text { de experimentar con métodos de investigación que propongo" }\end{array}$ \\
\hline
\end{tabular}




\begin{tabular}{|c|l|}
\hline 7 & $\begin{array}{l}\text { "...ir dando pasos más firmes y reales en la investigación documental ya } \\
\text { que se enfoca a una tarea específica" }\end{array}$ \\
\hline 8 & $\begin{array}{l}\text { "Ias evidencias propuestas en el programa: un estudio de caso y un } \\
\text { protocolo de investigación." }\end{array}$ \\
\hline 9 & $\begin{array}{l}\text { "En el ámbito epistemológico se logra que el alumno observe identificando } \\
\text { espacio tiempo en un texto narrativo...asimila la situación comunicativa en } \\
\text { significación. No logra dominar la interpretación, la aplicación, y no es muy } \\
\text { competente para transformar su experiencia lectora por medio de una } \\
\text { forma que no sea lingüística." }\end{array}$ \\
\hline
\end{tabular}

Las respuestas dadas a esta última pregunta son muy diversificadas. Como vemos algunas de ellas mencionan un porcentaje de avances (1 y 4 y 6 ), pero su referente no es el mismo; mientras que 1 se refiere a un porcentaje de avance en términos generales (suponemos que su parámetro es lo que el propio programa propone, el 4 se refiere al porcentaje de avance en relación al nivel con el que inician el curso, suponemos que hace un diagnóstico inicial, y el 6 se refiere al porcentaje de alumnos que "agarran la onda" y aplican en sus trabajos las sugerencias del profesor.

Los otras respuestas hacen alusión a diversos aspectos, así, algunas de ellas mencionan que tienen mayor claridad con respecto a lo que es realizar una investigación (2) o van dando pasos más firmes en el proceso (7), otras mencionan los productos que evidencian estos logros, tales como protocolos o informes (8), o las actividades que organizan para que los alumnos presente sus proyectos y avances (2).

Llama la atención la respuesta del informante 9, la cual enumera de forma detallada lo que sí logran los estudiantes y lo que se les dificulta, aunque se refiere exclusivamente a las habilidades lectoras. Por último, la respuesta que puede ser determinante en este rubro es la del informante 4, pues afirma que los logros son limitados, porque muy pocos alumnos se titulan mediante una tesis. 


\section{Conclusiones y recomendaciones}

El análisis de las respuestas de los profesores lleva a algunas reflexiones apenas concluyentes que abren la posibilidad a futuras indagaciones para profundizar en los ámbitos abiertos en este primer acercamiento:

Se puede ver que no existe un concepto común de "competencias investigativas" que unifique la práctica de los profesores, por lo que convendría un trabajo colegiado de conceptualización que sirva de base para la formación en la investigación.

La evaluación diagnóstica como práctica educativa necesaria en todo inicio de curso parece no ser común entre los profesores participantes. Y quienes dicen hacerla tampoco están unificados en el procedimiento.

Pareciera evidente que tampoco hay acuerdos entre los profesores en relación con la formación de competencias investigativas, por lo que convendría establecer hilos de articulación entre cada una de las etapas de la formación de estas competencias que den continuidad y solidez a esta formación.

Si cada uno de los profesores informantes ha identificado los aspectos más débiles de sus estudiantes respecto de las competencias investigativas, este aporte revela el potencial de intervención que pueden realizar en su práctica educativa para subsanarlas y consignarlas en los programas de asignatura.

De igual manera, con base en la información obtenida, se puede suponer que las "investigaciones" semestrales que realizan los estudiantes son parciales y responden más a lo que los docentes les solicitan en el curso escolar que a una investigación continua articulada en los distintos semestres de la formación investigativa. Por lo que es deseable ue ésta sea acompañada por los profesores, previo diálogo permanente y sistemático entre los docentes de la Academia para dar dirección y seguimiento a las incipientes investigaciones de los estudiantes.

Las respuestas de los profesores evidencian su preocupación respecto de que los estudiantes logren avances en su proceso formativo, aunque las acciones de diagnóstico, de fortalecimiento y 
de seguimiento no son muy sistemáticas y planificadas. Esto es posible a que las competencias investigativas y sus niveles de desarrollo no están claramente establecidas en los programas de asignatura.

Es de resaltar el hecho de que una de las deficiencias mencionadas por los profesores sea precisamente la dificultad para comprender conceptos teóricos, lo cual tiene que ver con las limitaciones para desarrollar un marco teórico, formular hipótesis y, en general, realizar cualquier actividad de análisis textual o simplemente para elaborar resúmenes. Esto es muy significativo, pues nos lleva a pensar en el bajo nivel de comprensión conceptual que tienen los estudiantes en el campo de la metodología para la investigación. Si a investigar se aprende investigando, la deficiencia de comprensión conceptual puede subsanarse con el acompañamiento docente en la elaboración de protocolos de investigación y la realización de la investigación de parte de los estudiantes.

También en el rubro de problemas se menciona la falta de tiempo como un factor que dificulta el desarrollo de las competencias investigativas. Efectivamente un semestre es muy poco tiempo para llevar a cabo un proceso de investigación completo. Esto podría ser causado por lo que se comentó en líneas anteriores con respecto a los programas de asignatura y la denominada Unidad de competencia, puesto que si no está especificado en el proyecto curricular cuál es nivel de desempeño o la unidad de competencia que debe lograse con cada una de las asignaturas, ni tampoco cuál o cuáles son las competencias a cuyo fomento contribuye la unidad de aprendizaje en cuestión ni el tiempo de su desarrollo, entonces el profesor puede asumir que debe atender todo el proceso investigativo en las sesenta horas clase que dura un semestre, lo cual es prácticamente imposible.

Por último, una de las respuestas que puede resultar más ilustrativa, en relación con el tema que aquí se trata, es la que afirma que los logros en este rubro son limitados, puesto que muy pocos alumnos se titulan mediante una tesis. Esto implicaría, por un lado, que los estudiantes no desarrollan de manera adecuada las competencias investigativas y por ello es que no se titulan 
mediante esta modalidad o no al no titularse por esta modalidad de tesis no desarrollan las competencias investigativas. Sin embargo, por otro lado, también podría pensarse que sí son competentes y saben hacer investigación, pero las otras opciones de titulación san más cómodas y fáciles que la de tesis. ¿Cuál será la realidad? Habrá qué indagar al respecto para afirmarlo con certeza.

La investigación es una de las actividades fundamentales en el programa de licenciatura de Letras hispánicas y es sabido que la mayoría de los docentes de dicha licenciatura realiza actividades de investigación. En este sentido, convendría investigar qué tanto y de qué manera estas actividades de investigación que realiza el docente impactan las actividades de enseñanza y aprendizaje, y de qué forma se involucra en ellas al alumno como parte de su proceso formativo.

Para finalizar, podemos afirmar que el egresado de Letras logra desarrollar algunas de las competencias investigativas mediante las habilidades y destrezas necesarias y el gusto para realizar investigaciones, sobre todo en el campo de la lengua y la literatura, de manera especial, en el campo del análisis literario y lingüístico, pero aún falta ampliar y profundizar las investigaciones en este campo para tener afirmaciones más contundentes acerca de su desempeño en el campo de la investigación. El binomio enseñanza/aprendizaje es complejo y si la complejidad sólo se entiende si se entienden los hilos que la entretejen, las perspectivas de los docentes son tan sólo un hilo en la complejidad de la formación de competencias investigativas.

\section{Referencias:}

Banderas, C., Cárdenas, G. y Martínez, E. (2013). La investigación formativa en el plan de estudios de la licenciatura en Letras hispánicas. Apuntes para un análisis de congruencia de los programas de asignatura. Revista Lápiz-cero. n. 6 p. 60-83. Recuperado de: https://issuu.com/aliciamartinezg/docs/revista I piz-cero 6

Guadarrama, P. (2014). Dirección y asesoría de la investigación científica. México: NEISA. 
Llano, A. (2003). Repensar la universidad: la universidad ante lo nuevo. Madrid: Ediciones Internacionales Universitarias.

Montero, A. (2009). Las competencias en educación. Competencias educativas, diseño y desarrollo del currículo en los centros. España: Guadalturia.

Moreno, M. G. (2005). Potenciar la educación. Un curriculum transversal de formación para la investigación. Revista Electrónica Iberoamericana sobre Calidad, Eficiencia y Cambio en Educación. 3(1) pp 520-540. Consultado el 15 de noviembre de 2017 en: http://www.redalyc.org:9081/articulo.oa?id=55130152

Muñoz, J. F., Quintero, J. y Múnevar, R. A. (2014). Cómo desarrollar competencias investigativas en educación. México: NEISA.

Navío, A. (2005). Las competencias profesionales del formador. Una visión desde la formación continua. Barcelona: Octaedro.

Núñez Cortés, J. A. (2016). “El modelo competencial y la competencia comunicativa en la educación superior en América Latina". Foro de Educación, 14(20), 467-488. doi: http://dx.doi.org/10.14516/fde.2016.014.020.023

Rojas, R. (2001). Formación de investigadores educativos. Una propuesta de investigación. México: Plaza y Valdés.

Sánchez, R. (2014). Enseñar a investigar Una didáctica nueva de la investigación en ciencias sociales y humanas. México: UNAM/IISUE.

Zabala, A. y Arnau, L. (2008). 11 ideas clave. Cómo aprender y enseñar competencias. Barcelona: GRAÓ. 